\title{
Late cardiac perconditioning by phenylephrine in an isolated rat heart model is mediated by mitochondrial potassium channels
}

\author{
Afshin Nazari' ${ }^{1}$, Mehrnoosh Sedighi², Parvin Dalvand ${ }^{1}$, Yaser Azizi ${ }^{3}$, Maryam Moghimian ${ }^{\circledR *}$, \\ Shakiba Nasiri Boroujeni²
}

\begin{abstract}
${ }^{1}$ Razi Herbal Medicines Research Center, Department of Physiology, Lorestan University of Medical Sciences, Khorramabad, Iran, ${ }^{2}$ Student Research Committee, Lorestan University of Medical Sciences, Khorramabad, Iran, ${ }^{3}$ Department of Physiology, School of Medicine, Iran University of Medical Sciences, Tehran, Iran, ${ }^{4}$ Department of Physiology, School of Medicine, Gonabad University of Medical Sciences, Gonabad, Iran
\end{abstract}

\begin{abstract}
The present study was designed to investigate the effect of early and late administration of phenylephrine during ischemia against regional ischemia-reperfusion injuries in an isolated rat heart model. All animals were randomly divided into experimental groups: (I) IR (Ischemic/ reperfusion): the hearts underwent $35 \mathrm{~min}$ of regional ischemia followed by $60 \mathrm{~min}$ of reperfusion; (II) $5 \mathrm{HD}-\mathrm{IR}-0$ : the hearts were perfused for 5 min with $5 \mathrm{HD}$ (5-hydroxydecanoate, specific mKATP channel blocker, $100 \mu \mathrm{M}$ ) at the onset of regional ischemia; (III) 5HD-IR-20: the hearts were perfused for 5 min with 5HD 20 min after regional ischemia; (IV) PE-IR-10: the hearts were perfused for $5 \mathrm{~min}$ with phenylephrine $10 \mathrm{~min}$ after regional ischemia; (V) PE-IR-30: the hearts were perfused for 5 min with phenylephrine (100 $\mu \mathrm{M}) 30$ min after regional ischemia; (VI) PE-5HD-IR-10 group: the hearts were perfused for 5 min with 5HD at the onset of regional ischemia after which phenylephrine was administrated as in group IV; and (VII) PE-5HD-IR-30: the hearts were perfused for $5 \mathrm{~min}$ with $5 \mathrm{HD} 20 \mathrm{~min}$ after the ischemia and then phenylephrine was administrated as in group V. The hemodynamic parameters were recorded throughout the experiment. Ischemia-induced arrhythmias, myocardial infarct size (IS), creatin kinase-MB isoenzyme (CK-MB), plasma lactate dehydrogenase (LDH) activities, and coronary blood flow (CBF) were measured in all animals. Perfusion of phenylephrine $30 \mathrm{~min}$ after the regional ischemia curtailed the myocardial infarct size, reduced CK-MB, and improved cardiac function and CBF. Administration of 5HD 30 min after the ischemia abolished cardioprotective effects of phenylephrine in the late phase. These results suggest the involvement of $\mathrm{mK}_{\text {ATP }}$ in the mechanism of phenylephrine-induced late preconditioning.
\end{abstract}

Keywords: Phenylephrine. Ischemia. Reperfusion. Preconditioning. Cardioprotection.

\section{INTRODUCTION}

Brief periods of ischemia lead to reduced severity of cardiac injury following a second sustained period of ischemia. This cardioprotective effect has been termed ischemic preconditioning (Tonkovic-Capin et al., 2002). Local preconditioning cannot be used in acute clinical settings such as acute myocardial infarction, ischemic stroke, or acute major vascular occlusion. It, therefore, has become necessary to develop new techniques suitable for providing protection against unpredictable ischemic events. One option is modifying the reperfusion period by means of brief coronary artery

\footnotetext{
*Correspondence: M. Moghimian. Department of Physiology, School of Medicine, Gonabad University of Medical Sciences, Gonabad, Iran. Tel: +98 9151778595 / Fax: +98 5157223814. E-mail: moghimian.m@gmu.ac.ir
}

occlusions and reperfusions applied at the onset of myocardial reperfusion, a phenomenon called ischemic postconditioning (IPOST). Although Na et al. (1996) formulated the seminal idea and terminology, the first easily reproducible experimental results on this topic were published by Zhao et al. (2003).

A shortcoming of both preconditioning and postconditioning is lengthened operative time, possibly even for duration of 15-20 min. A further negative aspect is that in the presence of atherosclerosis, these invasive techniques can lead to serious, life-threatening complications such as plaque rupture.

The role of $\alpha 1$-adrenoceptors has been studied extensively for the early preconditioning. Pharmacological activation of $\alpha 1$-adrenoceptors has been shown to mimic early preconditioning (Rojas Gomez et al., 2008; Salvi, 
2001) and its blockade nullifies the cardioprotective effect of preconditioning (Piascik, Perez, 2001).

Early studies have suggested that stimulation of $\mathrm{a}_{1}$-adrenoceptors is one of the essential triggers of the early phase of ischemic preconditioning (Banerjee et al., 1993). Also, exogenous activation of a1-adrenoceptor can exert both early (Imani et al., 2008; Salvi, 2001) and late preconditioning (Kudej et al., 2006; Tejero-Taldo et al., 2002). In those studies, the preconditioning stimulus was administered before the onset of myocardial ischemia.

Although the triggers and mediators of perconditioning are still not well understood, some studies have revealed that stimulation of $\alpha 1$-adrenoceptors is one of the essential triggers of the early phase of ischemic preconditioning (Banerjee et al., 1993). In addition, some studies have indicated that activation of $\alpha 1$-adrenoceptors can induce both early (Rojas Gomez et al., 2008; Salvi, 2001) and late preconditioning (Naderi et al., 2010b).

It has been shown that mitochondrial ATPsensitive K channels (mKATP) have a great influence on cardioprotection afforded by preconditioning (Rajesh et al., 2004; Tonkovic-Capin et al., 2002).

In this regard, diazoxide, as a specific opener of the mKATP channels, can mimic ischemic preconditioning (Shen et al., 2004), with application of 5HD (5-hydroxydecanoate), as a putatively specific mKATP channel blocker, preventing the cardioprotective effect of ischemic preconditioning (Tsukamoto et al., 2005) or pharmacological preconditioning. However, it has been suggested that the mKATP channels are involved as a subcellular mediator in preconditioning afforded by $\alpha 1$-adrenoceptor activation (Cohen et al., 2001; Gao, Chen, Yang, 2007), but the role of this channel in phenylephrineinduced late preconditioning is still unknown.

The present study was designed to investigate the effect of early and late administration of phenylephrine during ischemia against regional ischemia-reperfusion injuries in an isolated rat heart model. We also examined the role of the mKATP channels in this form of myocardial protection.

\section{MATERIAL AND METHODS}

\section{Preparation of Isolated Hearts}

Male Wistar rats (200-250 g) were housed in an air-conditioned colony room on a light/dark cycle at $21-23{ }^{\circ} \mathrm{C}$ with free access to food and water. The animals were anesthetized by sodium pentobarbital $(60 \mathrm{mg} / \mathrm{kg}$, i.p.) and given heparin sodium (500 IU). The hearts were rapidly excised and placed in ice-cold buffer, and mounted on a constant pressure $(80 \mathrm{mmHg}$ ) Langendorff-perfusion apparatus. All experiments were conducted in accordance with the institutional guidelines of Lorestan University of Medical Sciences (Khorramabad, Iran) as well as the National Institutes of Health guidelines for the care and use of laboratory animals. The hearts were perfused retrogradely with modified Krebs-Henseleit bicarbonate buffer containing (in mmol/L): $\mathrm{NaHCO} 325 ; \mathrm{KCl} 4.7$; $\mathrm{NaCl} 118.5 ; \mathrm{MgSO} 41.2 ; \mathrm{KH} 2 \mathrm{PO} 41.2$; glucose $11 ; \mathrm{CaCl} 2$ 2.5 gassed with $95 \% \mathrm{O} 2 / 5 \% \mathrm{CO} 2\left(\mathrm{pH} 7.35-7.45\right.$ at $37^{\circ} \mathrm{C}$ ). A latex, fluid-filled, isovolumic balloon was introduced into the left ventricle through the left atrial appendage and inflated to give a preload of 8 to $10 \mathrm{mmHg}$ while connected to a pressure transducer (Harvard, March-Hugsteten, Germany). Two thin stainless steel electrodes fixed at the ventricular apex and right atrium were employed to record ECG for monitoring the heart rate.

A surgical needle was passed under the origin of the left anterior descending coronary artery, while the ends of the suture were passed through a pipette tip to form a snare. Regional ischemia was induced by tightening the snare and reperfusion was performed by releasing the ends of the suture. The perfusion apparatus was water-jacketed to maintain a constant perfusion temperature of $37^{\circ} \mathrm{C}$. The hearts were allowed to beat spontaneously throughout the experiments. Hemodynamic parameters [left ventricular developed pressure and heart rate] were continuously monitored and recorded throughout the experiment using a computerized data acquisition system (ML750 Power Lab/4sp, AD Instruments) (Moghimian et al., 2014).

Left ventricular hemodynamic parameters including left ventricular end-diastolic pressure (LVEDP), left ventricular developed pressure [LVDP $=$ LVSP (left ventricular systolic pressure)-LVEDP], maximum rise and fall of $L V$ pressures $(+d p / d t$ and $-d p / d t$, respectively), and $\mathrm{RPP}($ rate pressure product $=\mathrm{LVDP} \times \mathrm{HR})$ were recorded at $10 \mathrm{~min}$ after termination of reperfusion. Also, coronary effluent was collected for CK-MB (Creatine KinaseMB) and LDH (lactate dehydrogenase) measurements.

\section{Experimental protocol}

Following heart isolation and prior to the baseline period, all hearts were perfused and allowed to stabilize for $30 \mathrm{~min}$ within which the heart rate and left ventricular developed pressure were maintained at the same level for three continuous measurement periods timed 5 min apart. The experimental design is illustrated in Figure 1. All animals were randomly divided into experimental groups ( $n=6-10$ ) as follows: (I) IR (Ischemic/ reperfusion) group: the hearts underwent $35 \mathrm{~min}$ of regional ischemia followed 
by 60 min reperfusion; (II) 5HD-IR-0 group: the hearts were perfused for 5 min with 5HD (5-hydroxydecanoate, specific mKATP channel blocker, $100 \mu \mathrm{M})$ at the onset of regional ischemia; (III) 5HD-IR-20 group: the hearts were perfused for 5 min with $5 \mathrm{HD}(100 \mu \mathrm{M}) 20$ min after regional ischemia; (IV) PE-IR-10 group: the hearts were perfused for 5 min with phenylephrine $(100 \mu \mathrm{M}) 10 \mathrm{~min}$ after regional ischemia; (V) PE-IR-30 group: the hearts were perfused for 5 min with phenylephrine $(100 \mu \mathrm{M})$ 30 min after regional ischemia; (VI) PE-5HD-IR-10 group: the hearts were perfused for $5 \mathrm{~min}$ with $5 \mathrm{HD}(100 \mathrm{mM})$ at the onset of regional ischemia and then phenylephrine was administrated as in group IV; (VII) PE-5HD-IR-30 group: the hearts were perfused for $5 \mathrm{~min}$ with $5 \mathrm{HD}(100 \mathrm{mM})$ 20 min after regional ischemia and then phenylephrine was administrated as in group $\mathrm{V}$.

Administrations of drugs in the early phase were performed via the second arm of perfusate cannula which was connected to the main perfusion cannula. The experimental conditions were constant throughout the experiment.

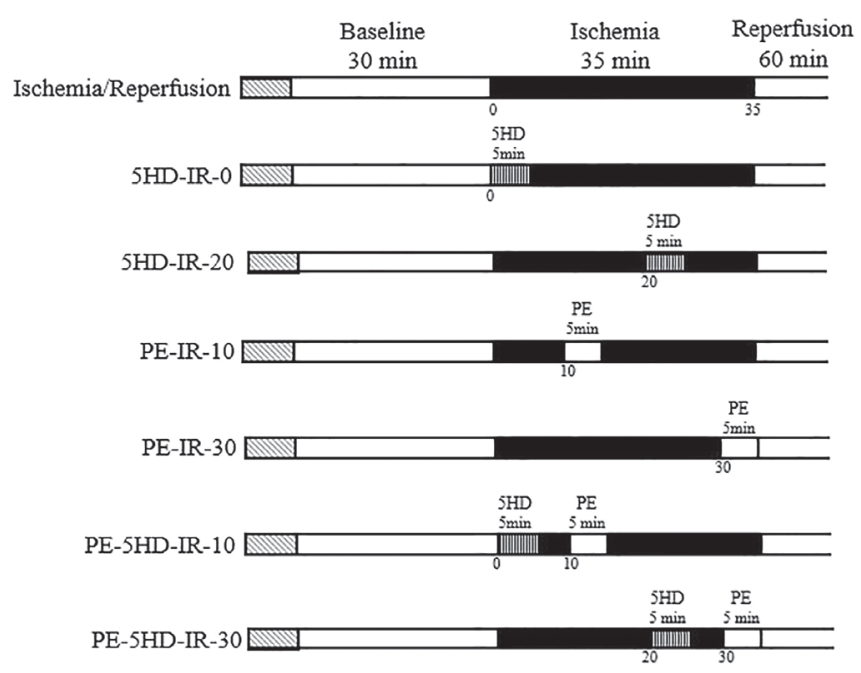

FIGURE 1 - Schematic illustration of the experimental groups: 5HD (5 hydroxydecanoate); PE (phenylephrine).

\section{Infarct size measurement}

Once the reperfusion period was completed, the hearts were frozen overnight and then sliced into 2-mm transverse sections from apex to base. The slices were then incubated with triphenyl tetrazolium chloride $1 \%$ (TTC in phosphate buffer $0.1 \mathrm{M}, \mathrm{pH}=7.4$ ) for a period of $20 \mathrm{~min}$ at $37^{\circ} \mathrm{C}$. TTC reacted with the viable tissue, producing a red formazan derivative, which was distinct from the white necrotic tissue once fixed in formalin $10 \%$ for $24 \mathrm{~h}$. The areas of the left ventricle and infracted tissues were measured through planimetry of the scanned hearts using Photoshop program. The volumes were obtained via multiplying the area by the thickness of the slices. The infarct size was expressed as the percentage of left ventricular volume for each heart (IS/LV).

\section{Determination of arrhythmia scores}

During the 30-min ischemia, ventricular arrhythmias were evaluated according to Lambeth convention (Walker et al., 1988).

Ventricular ectopic beats (VEBs) were defined as identifiable premature QRS complexes. Ventricular tachycardia (VT) was defined as a run of four or more ventricular premature beats. Finally, ventricular fibrillation (VF) was defined as the signal for which individual QRS deflections can no longer be distinguished from one other and the rate can no longer be measured. Original ECG recordings are illustrated in Figure 2. The onset time and duration of arrhythmias were captured to identify the severity of arrhythmias according to the following scoring system (Curtis, Walker, 1988). 0: $<10$ ventricular premature beats, $1: \geq 10$ ventricular premature beats, $2: \mathrm{VT}$ (duration $<30 \mathrm{~s}$ ), 3 : VT (duration $\geq 30 \mathrm{~s}$ ), 4 : VF starting 15 min after the onset of ischemia, 5: VF starting 5-15 min after the onset of ischemia, 6 : VF starting within $5 \mathrm{~min}$ after the onset of ischemia.
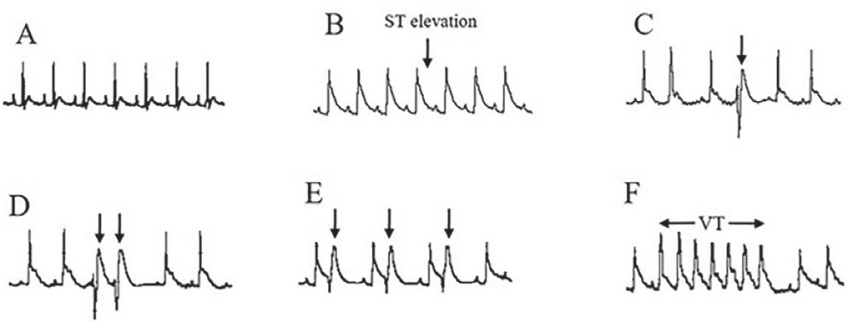

F
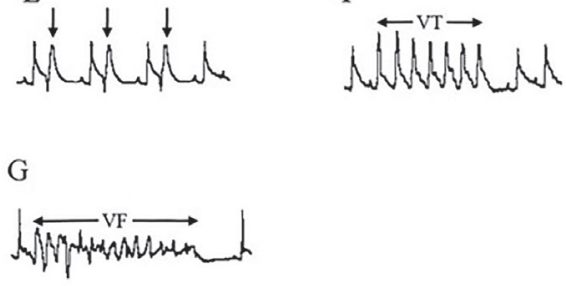

FIGURE 2 - Electrocardiogram recording; (A) During baseline; (B) during coronary artery occlusion; (C) ventricular ectopic beat (VEB); (D) couplet; (E) bigeminy; (F) ventricular tachycardia (VT); and (G) ventricular fibrillation (VF).

\section{Biochemical analysis}

The activity of CK-MB and LDH was calculated in coronary effluent samples at the end of reperfusion by commercial kits (Pars Azmoon, Iran) employing an autoanalyzer (Roche Hitachi Modular DP Systems, Mannheim, Germany). 


\section{Measuring the coronary blood flow}

The microvascular blood flow in the myocardium was recorded at the baseline, after $35 \mathrm{~min}$ of regional ischemia (ischemic myocardium), and after 60 minutes of reperfusion (reperfused myocardium), using laser Doppler flowmeter (ML191, Blood Flowmeter, AD Instruments). The data were expressed as the mean percentage of the baseline preischemia value.

\section{Chemicals}

Phenylephrine, 5HD, and triphenyltetrazolium chloride (TTC) were obtained from Sigma-Aldrich (Deisinhofen, Germany) and general laboratory chemicals were acquired from Merck (Darmstadt, Germany). Stock solutions of phenylephrine and 5HD were diluted with distilled water and added to the Krebs'-Henseleit bicarbonate (KHB) buffer and equilibrated with O2(95\%)$\mathrm{CO} 2(5 \%)\left(\mathrm{pH}=7.4\right.$ at $\left.37^{\circ} \mathrm{C}\right)$.

\section{Statistical analyses}

The data have been expressed as means \pm S.E.M. The statistical comparison of means between the groups was made by one-way ANOVA followed by Tukey test. The significant differences were determined as $\mathrm{P}<0.05$.

\section{RESULTS}

\section{Hemodynamic function}

The results in Table I indicate HR, LVDP, and rate pressure product (RPP as the percentage of an individual baseline). Since HR and LVDP may recover to different degrees, the rate pressure product was calculated through multiplying the heart rate by left ventricular developed pressure and presented as a reliable left ventricular function parameter for the isolated heart.

There were significant differences between RPP at the baseline and end of reperfusion $(p<0.05)$. The results revealed that administration of phenylephrine in phenylephrine-late perconditioning (PE-IR-30) group increased the recovery of RPP at the end of reperfusion in comparison with the IR group $(\mathrm{p}<0.05)$. The increased RPP by PE at late perconditioning was nullified by pretreatment with 5HD in 5HD-PE-IR-30 group as compared to PE-IR-30 $(\mathrm{p}<0.05)$.

\section{Infarct size and area at risk}

Figure 3 demonstrates the original pictures of TTC staining of the heart, with Figure 3B illustrating the ratio of the infarct size to the total left ventricular area. The ratio of infarct size to the total left ventricular area decreased considerably from $40.8 \pm 3.7$ in ischemia/ reperfusion group to $22.8 \pm 3.4$ in phenylephrine-late perconditioning (PE-IR-30) group. The reduction in the infarct size by $\mathrm{PE}$ at late perconditioning was nullified by pretreatment with 5HD in 5HD-PE-IR-30 group as compared to PE-IR-30 (35.4 $\pm 3 \%$ vs. $22.8 \pm 3.4 \%$ ). Our data analysis showed no significant differences in the infarct size between the other groups.

TABLE I - Hemodynamic parameters. HR (heart rate, beat per minute); LVDP (left ventricular developed pressure); RPP (rate pressure product) (beats/min $\mathrm{mmHg} \times 10^{3}$ ) in ischemia/reperfusion (IR), 5HD perfused at the onset of regional ischemia (5HDIR-0), 5HD perfused 20 min after regional ischemia (5HD-IR-20), phenylephrine-early perconditioning $10 \mathrm{~min}$ after ischemia (PE-IR-10), phenylephrine-late perconditioning $30 \mathrm{~min}$ after ischemia (PE-IR-30), 5HD-phenylephrine-early perconditioning (5HD-PE-IR-10), 5HD-phenylephrine-late perconditioning (5HD-PE-IR-30).

\begin{tabular}{|c|c|c|c|c|c|c|c|c|c|}
\hline \multirow[b]{2}{*}{ Group } & \multicolumn{3}{|c|}{ Baseline } & \multicolumn{3}{|c|}{ End of ischemia } & \multicolumn{3}{|c|}{ End of reperfusion } \\
\hline & $\begin{array}{c}\text { HR } \\
(\text { bpm) }\end{array}$ & $\begin{array}{l}\text { LVDP } \\
\text { mmHg }\end{array}$ & $\begin{array}{c}\text { RPP } \\
\text { bpm.mmHg }\end{array}$ & $\begin{array}{c}\text { HR } \\
\text { (bpm) }\end{array}$ & $\begin{array}{l}\text { LVDP } \\
\text { mmHg }\end{array}$ & RPP \% & $\begin{array}{c}\text { HR } \\
(\text { bpm) }\end{array}$ & $\begin{array}{l}\text { LVDP } \\
\text { mmHg }\end{array}$ & RPP \% \\
\hline$\overline{\mathrm{IR}}$ & $289 \pm 23$ & $82 \pm 5.1$ & $24057 \pm 3572$ & $273 \pm 19$ & $59 \pm 10$ & $42 \pm 8.8$ & $245 \pm 15^{\&}$ & $50 \pm 14^{\&}$ & $46 \pm 6.2^{\&}$ \\
\hline 5HD-IR-0 & $285 \pm 27$ & $99 \pm 17$ & $27279 \pm 4914$ & $264 \pm 24$ & $49.7 \pm 6$ & $48.8 \pm 10$ & $239 \pm 29^{\&}$ & $58.5 \pm 10.5^{\&}$ & $44 \pm 13.5^{\&}$ \\
\hline 5HD- IR-20 & $287 \pm 15.7$ & $90 \pm 6.3$ & $25919 \pm 773.1$ & $235 \pm 17.1$ & $39 \pm 4.6$ & $57 \pm 7.4$ & $246 \pm 17.4^{\&}$ & $50 \pm 3.1^{\&}$ & $48 \pm 1.4^{\&}$ \\
\hline PE- IR-10 & $290 \pm 28$ & $83 \pm 7.1$ & $24386 \pm 3290$ & $230 \pm 27$ & $63 \pm 11$ & $45 \pm 10.4$ & $239 \pm 24^{\&}$ & $75.5 \pm 5.4^{\&}$ & $65 \pm 7.9^{\&}$ \\
\hline PE- IR-30 & $300 \pm 15$ & $90 \pm 6.7$ & $27042 \pm 2726$ & $233 \pm 25$ & $70 \pm 6.9$ & $67 \pm 6.1$ & $230 \pm 24^{\&}$ & $71 \pm 9.8^{\&}$ & $78 \pm 3.8^{*} \#{ }^{*}$ \\
\hline 5HD-PE-IR-10 & $253 \pm 13.9$ & $89.2 \pm 7.8$ & $22478 \pm 1132$ & $212+9$ & $76 \pm 7.7$ & $57 \pm 6.8$ & $213.5 \pm 8.8^{\&}$ & $81 \pm 2.5^{\&}$ & $51.5 \pm 3.1^{\&}$ \\
\hline 5HD-PE-IR-30 & $266 \pm 28$ & $92 \pm 6$ & $24493 \pm 2635$ & $234 \pm 21$ & $70 \pm 16$ & $51.6 \pm 9.4$ & $205 \pm 12^{\&}$ & $68.5 \pm 7.3^{\&}$ & $47 \pm 10^{\&}$ \\
\hline
\end{tabular}

Repeated measure ANOVA was performed for inter- and intragroup comparisons. ${ }^{\circledR} \mathrm{P}<0.05$ vs. baseline. ${ }^{*} \mathrm{P}<0.05$ vs. IR group. ${ }^{\#}$ $\mathrm{P}<0.05$ vs. PE-5HD-IR-30 group. The data are presented as mean \pm SEM. 
A

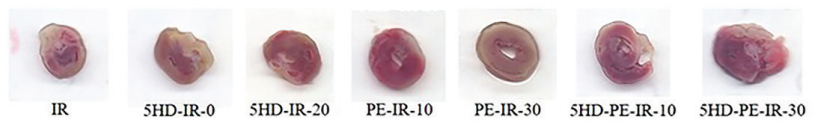

B

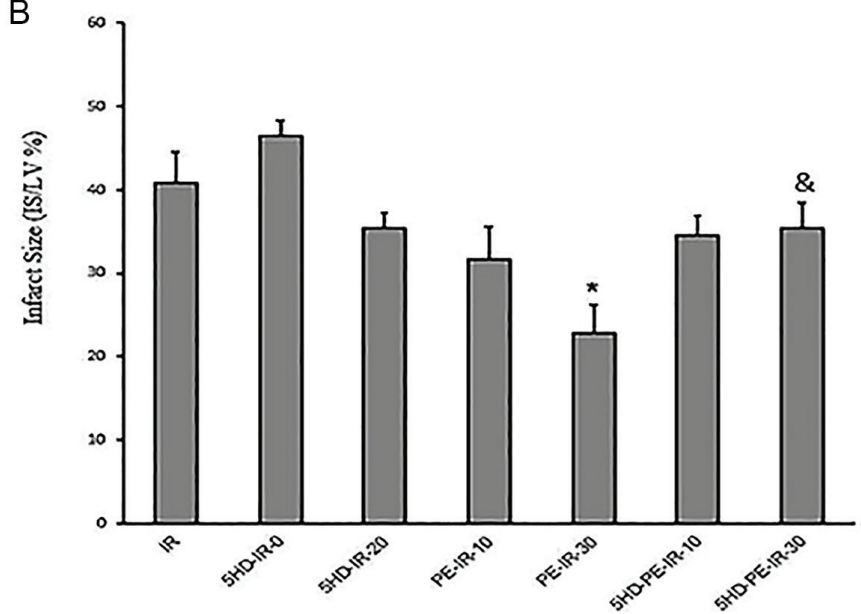

FIGURE 3 - A - The original pictures of TTC staining heart slices. 5HD, 5 hydroxydecanoate. B - The ratio of infarct size to total left ventricular area (IS/LV\%) in ischemia/reperfusion (IR), 5HD perfused at onset of regional ischemia (5HD-IR-0), 5HD perfused 20 min after regional ischemia (5HD-IR-20), phenylephrine-early perconditioning $10 \mathrm{~min}$ after ischemia (PE-IR-10), phenylephrine-late perconditioning $30 \mathrm{~min}$ after ischemia (PE-IR-30), 5HD-phenylephrine Early perconditioning (5HD-PE-IR-10), 5HD-phenylephrine-late perconditioning (5HD-PE-IR-30) groups. Data are presented as Mean \pm S.E.M. Significant difference with IR group $(\mathrm{P}<0.05)^{*}$. Significant difference with PE-IR-30 group $(\mathrm{P}<0.05){ }^{\text {\&. }}$.

\section{LDH and CK-MB activity}

LDH and CK-MB levels in the coronary artery effluent considerably declined by phenylephrine-induced perconditioning in the late phase (PE-IR-30) compared with ischemia/reperfusion group at $60 \mathrm{~min}(6.8 \pm 2.8$, $2.5 \pm 0.86$ versus $24 \pm 4.5,4.4 \pm 0.74$, respectively) after reperfusion. Addition of $5 \mathrm{HD}$ prior to $\mathrm{PE}$ restored the levels of LDH and CK-MB as seen in ischemia/ reperfusion group in 5HD-PE-IR-30 at $60 \mathrm{~min}(24.2 \pm 5.5$ and $4.7 \pm 0.8$ ) after reperfusion (Figure $4 \mathrm{~A}$ and $\mathrm{B}$ ). Our data analysis showed no significant differences in $\mathrm{LDH}$ and CK-MB levels between the other groups.

\section{Severity of arrhythmias}

Administration of PE, 30 min after the onset of ischemia (PE-IR-30) $(2 \pm 0.75)$ significantly reduced the severity of ventricular arrhythmias compared to IR $(4.2 \pm 1.1)$ and PE-IR-10 (3.85 \pm 0.67$)$ groups. Addition of 5HD prior to PE in 5HD-PE-IR-30 $(4 \pm 0.8)$ group
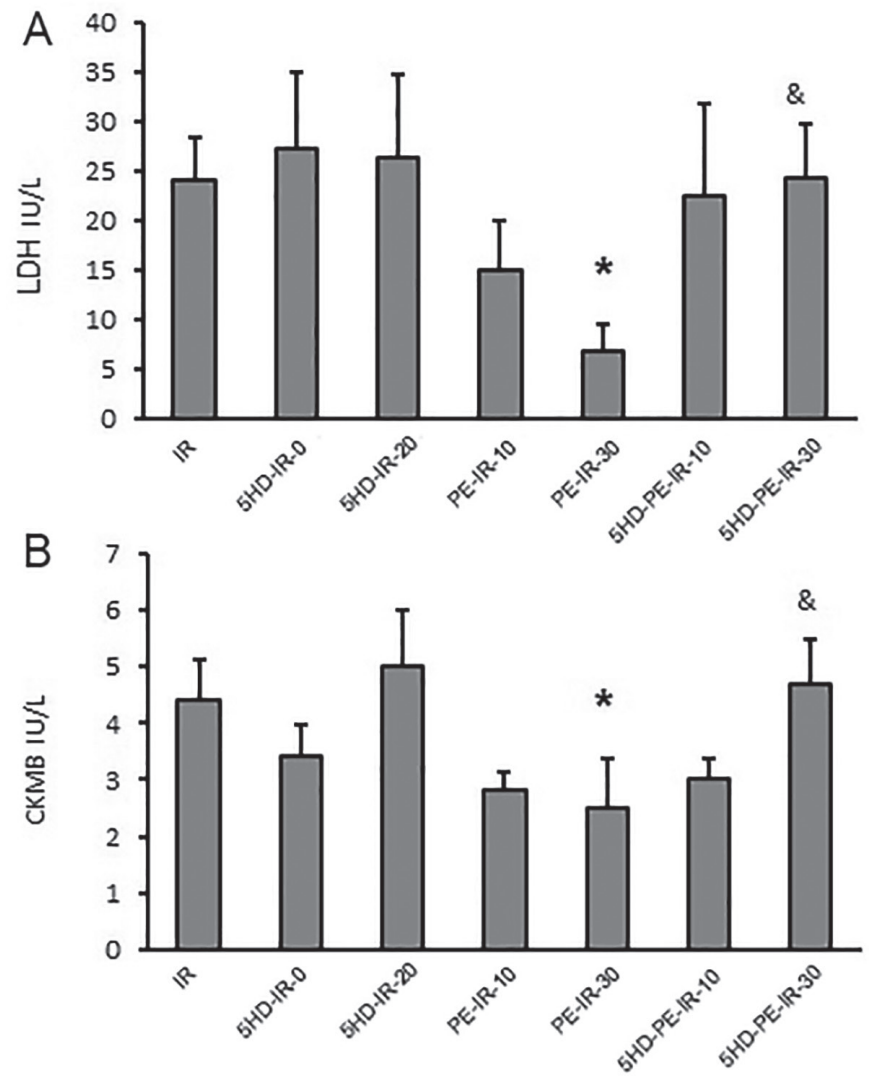

FIGURE 4 - A - Level of LDH and B - CK-MB in coronary effluent in $60 \mathrm{~min}$ after reperfusion in ischemia/reperfusion (IR), 5HD perfused at onset of regional ischemia (5HD-IR-0), 5HD perfused $20 \mathrm{~min}$ after regional ischemia (5HD-IR-20), phenylephrine-early perconditioning $10 \mathrm{~min}$ after ischemia (PE-IR-10), phenylephrine-late perconditioning $30 \mathrm{~min}$ after ischemia (PE-IR-30), 5HD-phenylephrine Early perconditioning (5HD-PE-IR-10), 5HD-phenylephrine-late perconditioning (5HD-PE-IR-30) groups. Data are presented as mean \pm SEM. Significant difference with IR group $(\mathrm{P}<0.05)^{*}$. Significant difference with PE-IR-30 group $(\mathrm{P}<0.05)$ \&.

intensified the severity of arrhythmias compared to PE-IR-30 (Figure 5).

\section{Number of PVC, VT and VF episodes}

The mean number of PVC episodes during $30 \mathrm{~min}$ of ischemia in the PE-IR-30 group $(16 \pm 6.6)$ was diminished considerably, compared with IR (63.8 \pm 13.8$)$ and PE-IR-10 (55 \pm 9$)$ groups. Addition of 5HD prior to $\mathrm{PE}$ in 5HD-PE-IR-30 (50 \pm 13.8$)$ enhanced the number of PVC episodes, as compared to PE-IR-30 (Figure 6A). Further, the administration of PE, 30 min after the onset of ischemia (PE-IR-30) $(0.14 \pm 0.14)$ significantly declined the number of VF episodes compared to IR $(2 \pm 0.9)$ group. Also, 5HD prior to PE in 5HD-PE-IR-30 (1.6 \pm 0.7$)$ group enhanced the number of VF compared to PE-IR-30 (Figure 


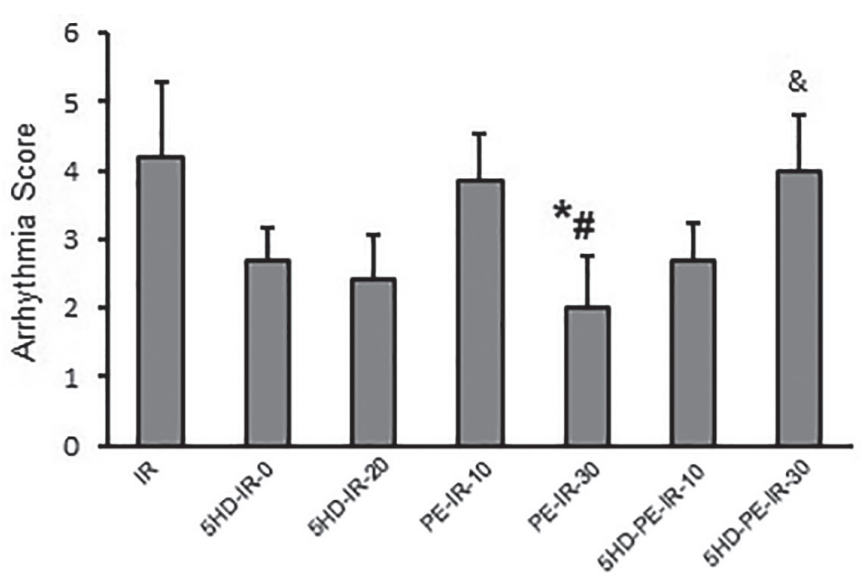

FIGURE 5 - Distribution of the arrhythmia score (severity) during $35 \mathrm{~min}$ ischemia in ischemia/reperfusion (IR), 5HD perfused at onset of regional ischemia (5HD-IR-0), 5HD perfused $20 \mathrm{~min}$ after regional ischemia (5HD-IR-20), phenylephrine-early perconditioning $10 \mathrm{~min}$ after ischemia (PE-IR-10), phenylephrine-late perconditioning $30 \mathrm{~min}$ after ischemia (PE-IR-30), 5HD-phenylephrine Early perconditioning (5HD-PE-IR-10), 5HD-phenylephrine-late perconditioning (5HD-PE-IR-30) groups. Data are presented as mean \pm SEM. " $\mathrm{P}<0.05$ vs. IR. ${ }^{\#} \mathrm{P}<0.05$ vs. PE-IR-10 group. ${ }^{\&} \mathrm{P}<0.05$ vs. PE-IR-30 group.

6C). There were no significant differences of the number of VT episodes between the experimental groups.

\section{Assessing coronary blood flow}

Coronary blood flow measured using LDF decreased by at least $60 \%$ after ischemia in all animals. Laser Doppler assessment of blood flow indicated a significant improvement of perfusion in the PE-IR-30 at the end of ischemia and reperfusion period. Addition of $5 \mathrm{HD}$ prior to PE lowered the LDF (Figure 7). There was no statistically significant difference in LDF between the other groups. There were no significant differences of BSC between the experimental groups either.

\section{DISCUSSION}

The present study indicated that perfusion of phenylephrine (as an $\alpha 1$-adrenoceptor agonist) in the late phase, $30 \mathrm{~min}$ after regional ischemia, decreased myocardial infarct size ( $\%$ of ischemia zone), reduced creatine kinase-MB (CK-MB) in the coronary effluent, and improved cardiac function. Administration of 5HD (5-hydroxydecanoate) as a putatively specific blocker of the mitochondrial KATP channels 30 min after the regional ischemia nullified the cardioprotective effects of phenylephrine in the late phase and brought the infarct size,
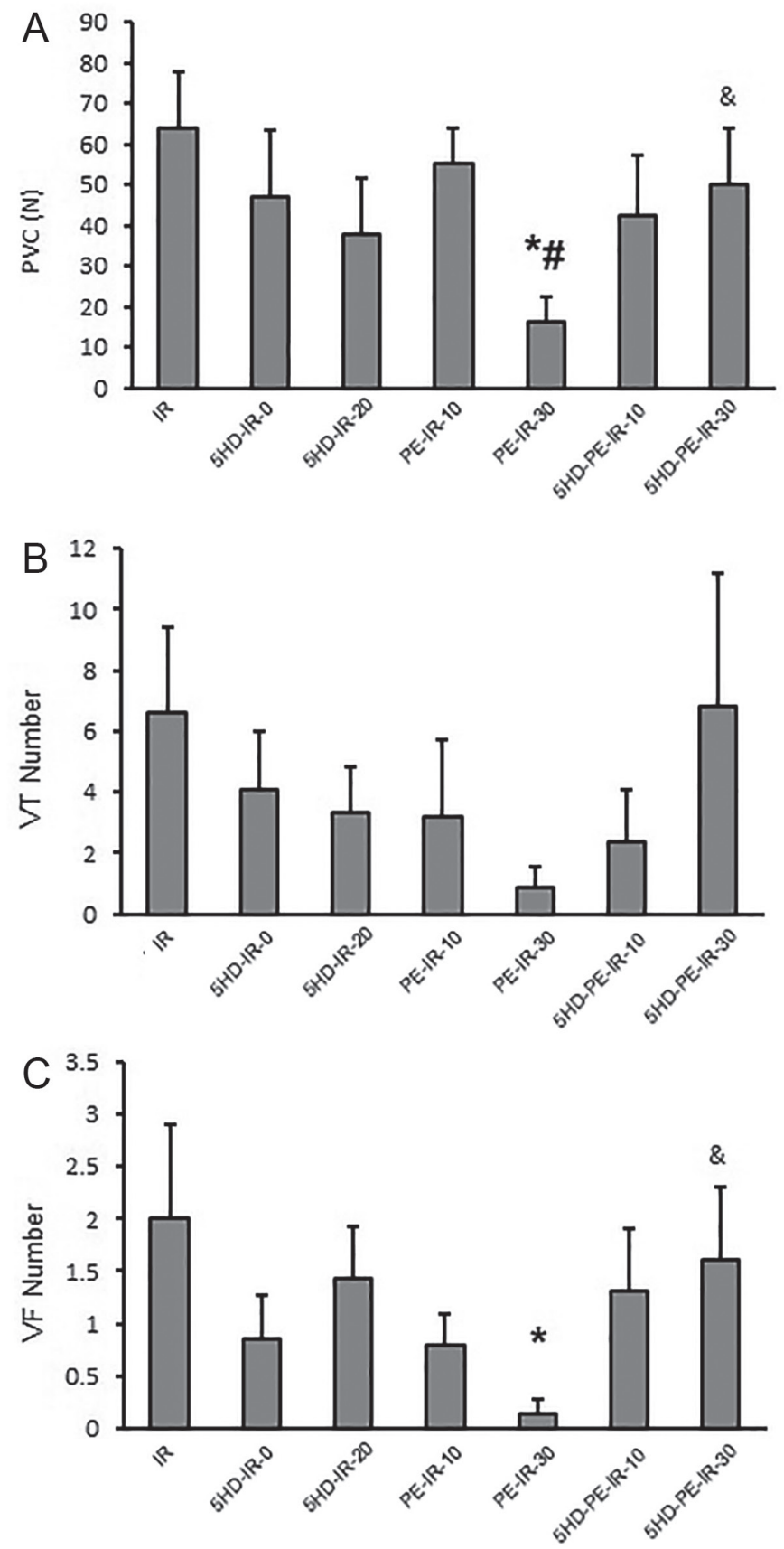

FIGURE 6 - A - episode number of PVC (premature ventricular contraction), B - episode number of VT (ventricular tachycardia) and $\mathrm{C}-\mathrm{VF}$ (ventricular fibrilation) during 30 min ischemia in ischemia/reperfusion (IR), 5HD perfused at onset of regional ischemia (5HD-IR-0), 5HD perfused 20 min after regional ischemia (5HD-IR-20), phenylephrineearly perconditioning $10 \mathrm{~min}$ after ischemia (PE-IR-10), phenylephrine-late perconditioning $30 \mathrm{~min}$ after ischemia (PE-IR-30), 5HD-phenylephrine Early perconditioning (5HDPE-IR-10), 5HD-phenylephrine-late perconditioning (5HD-PEIR-30) groups. Data are presented as mean $\pm \mathrm{SEM}$. ${ }^{*} \mathrm{P}<0.05$ vs IR. ${ }^{\#} \mathrm{P}<0.05$ vs. PE-IR-10 group. ${ }^{\&} \mathrm{P}<0.05$ vs. PE-IR-30 group. 


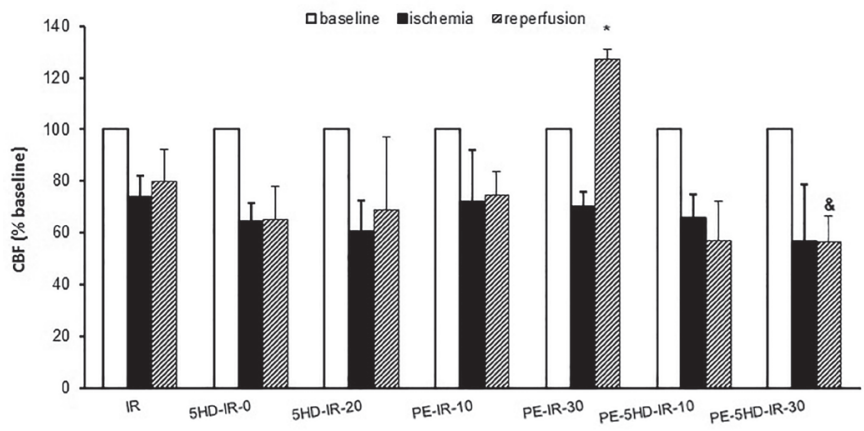

FIGURE 7 - The coronary blood flow (CBF) was recorded, at baseline, after $35 \mathrm{~min}$ of regional ischemia (ischemic myocardium), and after 60 minutes of reperfusion (reperfused myocardium), by using laser Doppler flowmeter LDF in ischemia/reperfusion (IR), 5HD perfused at onset of regional ischemia (5HD-IR-0), 5HD perfused 20 min of regional ischemia (5HD-IR-20), phenylephrine-early perconditioning $10 \mathrm{~min}$ of ischemia (PE-IR-10), phenylephrine-late perconditioning 30 min of ischemia (PE-IR-30), 5HD-phenylephrine Early perconditioning (5HD-PE-IR-10), 5HD-phenylephrine-late perconditioning (5HD-PE-IR-30). Repeated measure ANOVA was performed to compare between and within groups. * $\mathrm{P}<0.05$ vs. IR group $\& \mathrm{P}<0.05$ vs. PE-IR-30 group. Data were expressed as a mean percentage of the baseline preischemia value.

CK-MB and cardiac function to levels already observed in the ischemia/reperfusion group. Therefore, this result suggests involvement of $\mathrm{mK}_{\text {ATP }}$ in the mechanism of phenylephrine-induced late perconditioning.

In an early study, we also observed the contribution of mitochondrial ATP-sensitive potassium channel (mKATP) in protecting phenylephrine-induced early and late preconditioning in an isolated rat heart (Naderi, Imani, Faghihi, 2010a) .

The novelty of this study is that the $<$ alpha $>1$-adrenoceptor agonist was administered during regional ischemia (perconditioning model). Our study indicated that the pharmacological stimulation of the $\alpha 1$-adrenoceptors induced cardioprotection in the isolated rat heart when administered $30 \mathrm{~min}$ after the regional ischemia. However, applying pharmacological stimulation of the $\alpha 1$-adrenoceptors $10 \mathrm{~min}$ after the ischemia cannot induce the same effects. In the current experiment, phenylephrine administered $30 \mathrm{~min}$ after the onset of ischemia improved contractile function, reduced incidence of VT, and modified biochemical parameters in the same direction with the infarct size. We observed that phenylephrine after $30 \mathrm{~min}$ of ischemia attenuated heart function loss at the end of ischemia and reperfusion period, and caused a significant increase in RPP. The major effects of cardioprotection include reduced infarct size (anti-necrotic effect) (Ondrejcakova et al., 2009), lowered number and severity of cardiac arrhythmias (anti-arrhythmic effect) (Banerjee et al., 1993; Headrick, 1996), and improved contractile function (protection against contractile dysfunction) (Tsuchida et al., 1994). Elevated levels of CK have also been regarded as a specific biochemical marker of myocyte necrosis (Yilmaz et al., 2006). Further, LDH level plays an important role in systemic tissue damage (Devi et al., 2005). The effects of phenylephrine on releasing CK-MB and LDH were nullified by $5 \mathrm{HD}$. The differences between phenylephrine administration at 10 or 30 min after ischemia might be related to the influence of beta-receptor stimulation. The possible role of concentration, protein binding (95\%) and half-life of phenylephrine should also be considered. In addition, we observed that phenylephrine would stimulate microvascular blood flow in the myocardium and increase LDF, suggesting that its protect the myocardium from ischemia-reperfusion injury by preventing postischemic fall in blood flow. Enhancing blood flow to the myocardium is the aim of any form of treatment of ischemic heart disease.

Some experimental data have revealed that general activation starting $24 \mathrm{~h}$ after an ischemic event promotes the functional outcome without increasing tissue loss (Johansson, Ohlsson, 1996; Ohlsson, Johansson, 1995). One study indicated that preconditioning induced by intermittent limb ischemia administered after the onset of established myocardial ischemia and before reperfusion induced protection against myocardial dysfunction, malignant arrhythmia, and MI, through a KATP channeldependent mechanism (Schmidt et al., 2007). It is postulated that opening of mKATP could lead to $\mathrm{mPTP}$ inhibition via reducing mitochondrial $\mathrm{Ca}^{2+}$ load, enhancing mitochondrial energy production, or releasing ROS (Hausenloy et al., 2002).

It has also been demonstrated that there is a close relationship between the activity of Bcl-2 as an antiapoptotic protein and $\mathrm{MPTP}$ inhibition during late ischemic preconditioning (Rajesh et al., 2003). The inhibition of the mitochondrial permeability transition pore (mPTP) due to stimulation of $\alpha_{1}$-adrenoceptor is thought to be mediated by facilitated release of antiapoptotic proteins including Bcl-2 and activation of $\mathrm{mK}_{\mathrm{ATP}}$ channel (Naderi et al., 2010b).

In this study, the use of 5HD 30 min after the onset of ischemia rendered phenylephrine-induced cardioprotection ineffective in the late phase (late percondioning), suggesting that its effects are mediated, at least in part, by a KATP channel-dependent mechanism. Similarly, in our previous study, addition of 5HD reversed the protective effect of phenylephrine on hemodynamic 
parameters in early and late phases (Naderi, Imani, Faghihi, 2010a). These findings support the hypothesis that perconditioning utilizes a similar pathway, though experiments at the cellular level are required. As an example, under similar experimental settings, some experiments have shown that phenylephrine regulates mitochondrial membrane potential.

\section{CONCLUSIONS}

This study is the first to show that phenylephrine can induce late perconditioning via activating the $\mathrm{mK}_{\mathrm{ATP}}$ opening in an isolated rat heart.

\section{ACKNOWLEDGMENTS}

This study was supported by the grant of Tehran University of Medical Science.

\section{DISCLOSURES}

None of the authors have any competing interests.

\section{REFFERENCES}

Banerjee A, Locke-Winter C, Rogers KB, Mitchell MB, Brew EC, Cairns CB, Bensard DD, Harken AH. Preconditioning against myocardial dysfunction after ischemia and reperfusion by an a-1 adrenergic mechanism. Circ Res. 1993;73(4):656-70.

Cohen MV, Yang XM, Liu GS, Heusch G, Downey JM. Acetylcholine, bradykinin, opioids, and phenylephrine, but not adenosine, trigger preconditioning by generating free radicals and opening mitochondrial K(ATP) channels. Circ Res. 2001;89(3):273-278.

Curtis MJ, Walker MJ. Quantification of arrhythmias using scoring systems: an examination of seven scores in an in vivo model of regional myocardial ischaemia. Cardiovasc Res. 1988;22(9):656-665.

Devi R, Banerjee SK, Sood S, Dinda AK, Maulik SK. Extract from Clerodendron colebrookianum Walp protects rat heart against oxidative stress induced by ischemic-reperfusion injury (IRI). Life Sci. 2005;77(24):2999-3009.

Gao H, Chen L, Yang HT. Activation of alpha1B-adrenoceptors alleviates ischemia/reperfusion injury by limitation of mitochondrial $\mathrm{Ca} 2+$ overload in cardiomyocytes. Cardiovasc Res. 2007;75(3):584-595.
Hausenloy DJ, Maddock HL, Baxter GF, Yellon DM. Inhibiting mitochondrial permeability transition pore opening: a new paradigm for myocardial preconditioning? Cardiovasc Res. 2002;55(3):534-543.

Headrick JP. Ischemic preconditioning: bioenergetic and metabolic changes and the role of endogenous adenosine. J Mol Cell Cardiol. 1996;28(6):1227-40.

Imani A, Faghihi M, Sadr SS, Keshavarz M, Niaraki SS. Noradrenaline reduces ischemia-induced arrhythmia in anesthetized rats: involvement of alpha1-adrenoceptors and mitochondrial K ATP channels. J Cardiovasc Electrophysiol. 2008;19(3):309-315.

Johansson BB, Ohlsson AL. Environment, social interaction, and physical activity as determinants of functional outcome after cerebral infarction in the rat. Exp Neurol. 1996;139(2):322-327.

Kudej RK, Shen YT, Peppas AP, Huang CH, Chen W, Yan L, Vatner DE, Vatner SF. Obligatory role of cardiac nerves and alpha1-adrenergic receptors for the second window of ischemic preconditioning in conscious pigs. Circ Res. 2006;99(11):127076.

Moghimian M, Faghihi M, Karimian SM, Imani A, Mobasheri MB. Upregulated Hsp27 expression in the cardioprotection induced by acute stress and oxytocin in ischemic reperfused hearts of the rat. Chin J Physiol. 2014;57(6):329-34.

Na HS, Kim YI, Yoon YW, Han HC, Nahm SH, Hong SK. Ventricular premature beat-driven intermittent restoration of coronary blood flow reduces the incidence of reperfusioninduced ventricular fibrillation in a cat model of regional ischemia. Am Heart J. 1996;132(1 Pt 1):78-83.

Naderi R, Imani A, Faghihi M. Phenylephrine produces late pharmacological preconditioning in the isolated rat heart. Eur J Pharmacol. 2010a;627(1-3):203-8.

Naderi R, Imani A, Faghihi M, Moghimian M. Phenylephrine induces early and late cardioprotection through mitochondrial permeability transition pore in the isolated rat heart. J Surg Res. 2010b;164(1):e37-42.

Ohlsson AL, Johansson BB. Environment influences functional outcome of cerebral infarction in rats. Stroke. 1995;26(4):64449. 
Ondrejcakova M, Ravingerova T, Bakos J, Pancza D, Jezova D. Oxytocin exerts protective effects on in vitro myocardial injury induced by ischemia and reperfusion. Can J Physiol Pharmacol. 2009;87(2):137-42.

Piascik MT, Perez DM. Alpha1-adrenergic receptors: new insights and directions. J Pharmacol Exp Ther. 2001;298(2):40310.

Rajesh KG, Sasaguri S, Suzuki R, Xing Y, Maeda H. Ischemic preconditioning prevents reperfusion heart injury in cardiac hypertrophy by activation of mitochondrial KATP channels. Int J Cardiol. 2004;96(1):41-49.

Rajesh KG, Sasaguri S, Zhitian Z, Suzuki R, Asakai R, Maeda $\mathrm{H}$. Second window of ischemic preconditioning regulates mitochondrial permeability transition pore by enhancing Bcl-2 expression. Cardiovasc Res. 2003;59(2):297-307.

Rojas Gomez DM, Schulte JS, Mohr FW, Dhein S. Alpha1 -adrenoceptor subtype selective regulation of connexin 43 expression in rat cardiomyocytes. Naunyn Schmiedebergs Arch Pharmacol. 2008;377(1):77-85.

Salvi S. Protecting the myocardium from ischemic injury: a critical role for alpha(1)-adrenoreceptors? Chest. 2001;119(4):1242-9.

Schmidt MR, Smerup M, Konstantinov IE, Shimizu M, Li J, Cheung $\mathrm{M}$, et al. Intermittent peripheral tissue ischemia during coronary ischemia reduces myocardial infarction through a KATP-dependent mechanism: first demonstration of remote ischemic perconditioning. Am J Physiol Heart Circ Physiol. 2007;292(4):H1883-90.

Shen YL, Chen YY, Wu XD, Bruce IC, Xia Q. Activation of mitochondrial ATP-sensitive potassium channels delays ischemia-induced cellular uncoupling in rat heart. Acta Pharmacol Sin. 2004;25(1):22-8.
Tejero-Taldo MI, Gursoy E, Zhao TC, Kukreja RC. Alphaadrenergic receptor stimulation produces late preconditioning through inducible nitric oxide synthase in mouse heart. J Mol Cell Cardiol. 2002;34(2):185-95.

Tonkovic-Capin M, Gross GJ, Bosnjak ZJ, Tweddell JS, Fitzpatrick CM, Baker JE. Delayed cardioprotection by isoflurane: role of K(ATP) channels. Am J Physiol Heart Circ Physiol. 2002;283(1):H61-8.

Tsuchida A, Liu Y, Liu GS, Cohen MV, Downey JM. alpha 1 -adrenergic agonists precondition rabbit ischemic myocardium independent of adenosine by direct activation of protein kinase C. Circ Res. 1994;75(3):576-85.

Tsukamoto O, Asanuma H, Kim J, Minamino T, Takashima S, Ogai A, et al. A role of opening of mitochondrial ATP-sensitive potassium channels in the infarct size-limiting effect of ischemic preconditioning via activation of protein kinase $\mathrm{C}$ in the canine heart. Biochem Biophys Res Commun. 2005;338(3):1460-6.

Walker MJ, Curtis MJ, Hearse DJ, Campbell RW, Janse MJ, Yellon DM, et al. The Lambeth Conventions: guidelines for the study of arrhythmias in ischaemia infarction, and reperfusion. Cardiovasc Res. 1988;22(7):447-55.

Yilmaz A, Yalta K, Turgut OO, Yilmaz MB, Ozyol A, Kendirlioglu O, et al. Clinical importance of elevated CK-MB and troponin I levels in congestive heart failure. Adv Ther. 2006;23(6):1060-7.

Zhao ZQ, Corvera JS, Halkos ME, Kerendi F, Wang NP, Guyton RA, Vinten-Johansen J. Inhibition of myocardial injury by ischemic postconditioning during reperfusion: comparison with ischemic preconditioning. Am J Physiol Heart Circ Physiol. 2003;285(2):H579-88.

Received for publication on $06^{\text {th }}$ February 2018 Accepted for publication on $09^{\text {th }}$ August 2018 